\title{
Thermophoresis and photophoresis of cylindrical particles
}

\author{
Huan J. Keh *, Hung J. Tu \\ Department of Chemical Engineering, National Taiwan University, Taipei, 106-17 Taiwan, ROC
}

Received 7 June 1999; accepted 20 March 2000

\begin{abstract}
An analytical study is presented for the thermophoresis and photophoresis of a cylindrical aerosol particle in the direction normal to its axis. The Knudsen number is assumed to be small so that the fluid flow is described by a continuum model with a temperature jump, a thermal slip, and a frictional slip at the particle surface. In the limit of small Peclet and Reynolds numbers, the energy and momentum equations governing these problems are solved at steady state. Expressions for the migration velocity of the particle are obtained in simple closed forms for the cases of thermophoresis in a uniformly prescribed bulk-gas temperature gradient and of photophoresis subjected to an intense light beam. The results indicate that the thermophoretic mobility of a cylindrical particle is smaller than that of a sphere, keeping the properties of the gas and particle unchanged. On the other hand, the photophoretic mobility of a cylindrical particle can be greater or smaller than that of a sphere, depending on the ratio of particle-to-gas thermal conductivities and the relative magnitude of the temperature jump coefficient at the gas/particle interface. (C) 2001 Elsevier Science B.V. All rights reserved.
\end{abstract}

Keywords: Thermophoresis; Photophoresis; Aerosol cylinders

\section{Introduction}

The problem of slow relative motions between a rarefied gas and a small particle has received considerable attention in the general investigations of aerosol mechanics. However, the gasparticle systems were traditionally assumed to be isothermal. It is well known that a temperature gradient along the surface of a solid particle can cause a thin interfacial layer of gas to move in the direction of the gradient. This phenomenon,

\footnotetext{
* Corresponding author. Tel.: + 886-2-23635462; fax: + 886-2-23623040.

E-mail address: huan@ccms.ntu.edu.tw (H.J. Keh).
}

termed 'thermal slip,' was first examined by Maxwell [1] using gas kinetic theory. The thermal slip over a particle in the continuum regime (the Knudsen number $l / a \ll 1$, where $l$ is the mean free path of the gas molecules and $a$ is the radius of the particle) provides mechanisms for the thermophoresis and photophoresis of aerosol particles. In thermophoresis, the temperature inhomogeneity at the surface of the particle is due to a prescribed gradient in the carrier gas, while in photophoresis no temperature gradient in the bulk gas is maintained and the temperature gradient along the particle surface originates from a nonuniform absorption of the radiant energy by the particle. 
The thermophoretic and photophoretic effects can be explained in part by appealing to the kinetic theory of gases [2]. The higher energy molecules in the hot regions of the gas impinge on the particle with greater momenta than molecules coming from the cold regions, thereby leading to the migration of the particle in the direction opposite to the surface temperature gradient. Thus, for thermophoresis, the particle always moves in the direction of decreasing bulk-gas temperature. However, the photophoretic force on an aerosol particle can be directed either toward (negative photophoresis) or away from (positive photophoresis) the light source, depending upon the optical characteristics of the particle. If the particle is opaque and the incident light energy is adsorbed and dissipated directly at the front surface of the particle, positive photophoresis occurs. Conversely, if the light beam is partially transmitted and focused at some other internal area (e.g. the rear surface) of the particle, motion in other directions may result.

Deposition by thermophoresis is of considerable practical importance in many industrial applications when hot gases containing small suspended particles flow over cool surfaces. For example, thermophoresis can be effective in removing or collecting small particles from laminar gas streams in air cleaning and aerosol sampling devices [3]. The phenomenon has also been cited as an origin for the deposition of particulate matter on surfaces of heat exchangers causing scale formation with the attendant reduction of the heat-transfer coefficient [4]. Convincing evidence has been provided that, in the modified chemical vapor deposition process for the manufacture of high quality optical fibers, thermophoresis is the primary mechanism responsible for the deposition of aerosol particles (soot) onto the inner walls of the containing tube [5]. On the other hand, deposition of contaminant particles by thermophoresis on wafers in clean rooms during manufacturing steps can be a major cause of loss of product yields in the microelectronics industry [6]. In the area of nuclear safety, knowledge of thermophoresis is required to calculate the deposition rates of radioactive aerosol particles released in reactor accident situations where large temperature gradients exist [7].
Since photophoresis is observed for many particulate materials in the diameter range between $10^{-8}$ and $10^{-3} \mathrm{~m}$, and at pressures from above 1 atm down to below 1 torr, under illumination intensities comparable with sunlight [8], the results of photophoresis investigations are of interest to a wide variety of fields including cloud physics, aerosol science, and environmental engineering. For example, measurements of the photophoretic force or the reversal point from positive to negative photophoresis with the elaboration of photophoretic spectroscopy can be used to determine the physical properties, such as the complex refractive index, and the chemical composition of aerosol particles $[9,10]$. The photophoretic phenomena of aerosol particles subjected to coherent light beams have been applied to the development of laser atmospheric monitoring methods [11]. It was found that, due to the effect of both positive and negative photophoresis, stratospheric aerosol particles may be caused to rise against the gravity while others are induced to fall considerably more rapidly than they would under gravity alone $[12,13]$. Considering that radiative transfer can account for around $95 \%$ of the total heat flux in pulverized-coal furnaces, the driving force for photophoresis of small particles in combustion environments can be significantly greater than that for thermophoresis [14].

Standard theoretical work on thermophoresis and photophoresis is limited to spherical particles. Based on the assumptions of a small Knudsen number, small Peclet number, and small Reynolds number, as well as the effects of temperature jump, thermal slip, and frictional slip at the gas/ particle interface, Brock [15] determined the thermophoretic velocity of an aerosol sphere in a constant temperature gradient, while Reed [16] and Mackowski [14] obtained the photophoretic velocity of a spherical particle illuminated by an intense light beam. In the free molecule regime $(l / a \gg 1)$, the velocity distribution of the incoming gas molecules may be taken to be uninfluenced by the small particle and given by the MaxwellBoltzmann distributions. Under this assumption, well-established models are available for the thermophoretic velocity [17-19] and photophoretic velocity $[20,21]$ of a spherical particle. 
Of course, real aerosol particles are seldom perfect spheres. In the continuum regime, the extension of Brock's [15] analysis to the thermophoresis of a nonspherical particle has only been made for the case of axisymmetric migration of a prolate or oblate spheroid, without considering the effects of temperature jump and frictional slip at the gas/particle interface [7,22]. Recently, the photophoresis of an aggregate of two spheres [23] and of an arbitrarily shaped particle [24] has also been examined to some extent. In this paper we present an analytical study of the thermophoretic and photophoretic motions of a circular cylindrical particle in the direction normal to its axis in the continuum regime. The energy and momentum equations applicable to the system are solved by satisfying the conditions of temperature jump, thermal slip and frictional slip at the gas/ particle interface. The particle velocities are determined in simple closed forms as a function of relevant parameters.

\section{$<$ sbm 42>2. Thermophoresis of a cylinder}

In this section we consider the steady thermophoretic motion of a long cylindrical particle of radius $a$ with internal heat conductivity $k_{\mathrm{p}}$ in an unbounded gaseous medium where the heat conductivity is $k$. The cylinder is oriented perpendicularly to the uniformly imposed temperature gradient $-E_{\infty} \mathbf{e}_{x}$ (equal to $\nabla T_{\infty}$ ), as shown in Fig.

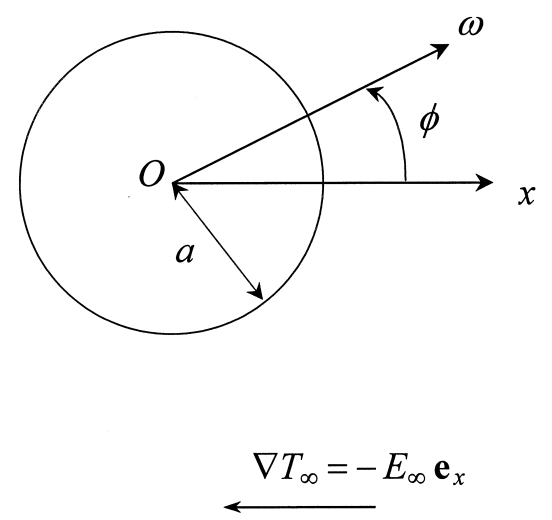

Fig. 1. Geometrical sketch for the two-dimensional thermophoretic motion of a cylinder.
1 , where $\mathbf{e}_{x}$ is the unit vector in the $x$ direction. It is assumed that $E_{\infty} a / T_{0} \ll 1$, where $T_{0}$ is the prescribed temperature at the axis of the cylinder (or the mean gas temperature in the vicinity of the particle). All the physical properties of the particle and the fluid are taken to be constant, and the Knudsen number, Reynolds number, and Peclet number are assumed to be small. Gravitational effects are ignored. Our objective is to obtain the thermophoretic velocity of the cylinder.

It is well known that in transverse motion end effects modify the temperature distribution and flow pattern at large distance from the axis of the cylinder. However, near the cylinder and not too close to the ends, the heat transfer and fluid flow are essentially perpendicular to the cylinder axis. Hence, it may be treated as a two-dimensional problem in a cross section perpendicular to the cylinder. Because the boundary conditions of the fluid velocity field are coupled with the temperature gradient at the particle surface, it is necessary to determine the temperature profile first.

\section{$<$ sbm 42>SBM 18>2.1. Temperature distribution}

The energy equations governing the temperature distribution are:

$\nabla^{2} T=0$

for the fluid and

$\nabla^{2} \hat{T}=0$

for the cylindrical particle. In the polar coordinate system $(\omega, \phi)$ measured from the axis of the cylinder,

$\nabla^{2}=\frac{1}{\omega} \frac{\partial}{\partial \omega}\left(\omega \frac{\partial}{\partial \omega}\right)+\frac{1}{\omega^{2}} \frac{\partial^{2}}{\partial \phi^{2}}$.

The boundary conditions at the particle surface $(\omega=a)$ require that the normal heat fluxes be continuous and a temperature jump which is proportional to the normal temperature gradient [2] occur. Also, the fluid temperature must approach the linear prescribed field far from the particle and the temperature inside the particle is finite everywhere. Thus, one has: 
$\omega=a: \quad k \frac{\partial T}{\partial \omega}=k_{\mathrm{p}} \frac{\partial \hat{T}}{\partial \omega}$

$\omega=a: \quad T-\hat{T}=C_{\mathrm{t}} \mathrm{l} \frac{\partial T}{\partial \omega}$,

$\omega<a: \quad \hat{T}$ is finite,

$\omega \rightarrow \infty: \quad T \rightarrow T_{\infty}=T_{0}-E_{\infty} \omega \cos \phi$.

Here, $C_{\mathrm{t}}$ is the dimensionless temperature jump coefficient (of order unity) which is semi-empirically related to the thermal accommodation coefficient $f_{\mathrm{t}}$ at the particle surface by $C_{\mathrm{t}} \cong(15 / 8)\left(2-f_{\mathrm{t}}\right) / f_{\mathrm{t}}$ and $l$ is the mean free path of the surrounding gas. The thermal accommodation coefficient is defined by $f_{\mathrm{t}}=\left(E_{\mathrm{i}}-E_{\mathrm{r}}\right) /\left(E_{\mathrm{i}}-E_{\mathrm{w}}\right)$, where $E_{\mathrm{i}}$ represents the average incident molecular energy flux at a point on the surface, $E_{\mathrm{r}}$ denotes the average reflected energy flux at that point, and $E_{\mathrm{w}}$ is the energy flux which would be emitted if the gas molecules left in thermal equilibrium with the surface at that point $[2,17]$.

The solution to Eqs. $(1 \mathrm{a}, \mathrm{b})$ and Eqs. $(3 \mathrm{a}-\mathrm{d})$ is:

$T=T_{0}-E_{\infty}\left[1+\frac{1-k^{*}+k^{*} C_{\mathrm{t}}^{*}}{1+k^{*}+k^{*} C_{\mathrm{t}}^{*}}\left(\frac{a}{\omega}\right)^{2}\right] \omega \cos \phi$,

$\hat{T}=T_{0}-\frac{2}{1+k^{*}+k^{*} C_{\mathrm{t}}^{*}} E_{\infty} \omega \cos \phi$,

where

$k^{*}=k_{\mathrm{p}} / k$,

$C_{\mathrm{t}}^{*}=C_{\mathrm{t}} l / a$.

It is understood that the practical value of $C_{\mathrm{t}}^{*}$ is less than unity with the assumption of a small Knudsen number. Note that the dimensionless parameter $k^{*} C_{t}^{*}$ denotes the relative resistance caused by the temperature jump at the particle surface with respect to the heat conduction inside the particle.

\section{$<$ sbm $42>2.2$. Fluid velocity distribution}

With knowledge of the solution for the temperature distribution, we can now proceed to find the fluid velocity field. The fluid surrounding the particle is assumed to be incompressible and Newtonian. Because the Reynolds number is small, the fluid motion caused by the thermophoretic migration of the cylinder is governed by the fourth-order linear differential equation for the stream function $\Psi(\omega, \phi)$,

$\nabla^{4} \Psi=\nabla^{2}\left(\nabla^{2} \Psi\right)=0$.

The stream function is related to the $\omega$ and $\phi$ components of the fluid velocity by:

$v_{\omega}=\frac{1}{\omega} \frac{\partial \Psi}{\partial \phi}$,

$v_{\phi}=-\frac{\partial \Psi}{\partial \omega}$.

Owing to the thermal creep and frictional slip velocities along the solid/fluid interface [15] as well as the fluid at rest far from the particle, the boundary conditions for the fluid velocity are:

$\omega=a: \quad v_{\omega}=U \cos \phi$,

$\omega=a: \quad v_{\phi}=-U \sin \phi+\frac{C_{\mathrm{m}} l}{\eta} \tau_{\omega \phi}+\frac{C_{\mathrm{s}} \eta}{\rho T_{0}} \omega \frac{\partial T}{\partial \phi}$,

$\omega \rightarrow \infty: \quad v_{\omega} \rightarrow 0$,

$\omega \rightarrow \infty: \quad v_{\phi} \rightarrow 0$.

Here, the polar coordinate system $(\omega, \phi)$ originated from the axis of the cylinder is fixed with the laboratory reference frame, $\rho$ and $\eta$ are the density and viscosity, respectively, of the gas, $C_{\mathrm{m}}$ is the dimensionless coefficient of the gas-kinetic isothermal slip which is semi-empirically related to the momentum accommodation coefficient $f_{\mathrm{m}}$ at the particle/fluid interface by $C_{\mathrm{m}} \cong\left(2-f_{\mathrm{m}}\right) / f_{\mathrm{m}}$, $C_{\mathrm{s}}$ is the dimensionless thermal creep coefficient about the particle surface (both $C_{\mathrm{m}}$ and $C_{\mathrm{s}}$ are of order unity), $\tau_{\omega \phi}$ is the shear stress for the fluid flow,

$\tau_{\omega \phi}=\eta\left[\omega \frac{\partial}{\partial \omega}\left(\frac{v_{\phi}}{\omega}\right)+\frac{1}{\omega} \frac{\partial v_{\omega}}{\partial \phi}\right]$,

and $U$ is the thermophoretic velocity of the cylinder (in the $x$ direction) to be determined.

The momentum accommodation coefficient is defined by $f_{\mathrm{m}}=\left(G_{\mathrm{i}}-G_{\mathrm{r}}\right) / G_{\mathrm{i}}$, where $G_{\mathrm{i}}$ and $G_{\mathrm{r}}$ are the average tangential components of the momentum of the gas molecules hitting and leaving the surface, respectively [2,17]. A set of reasonable 


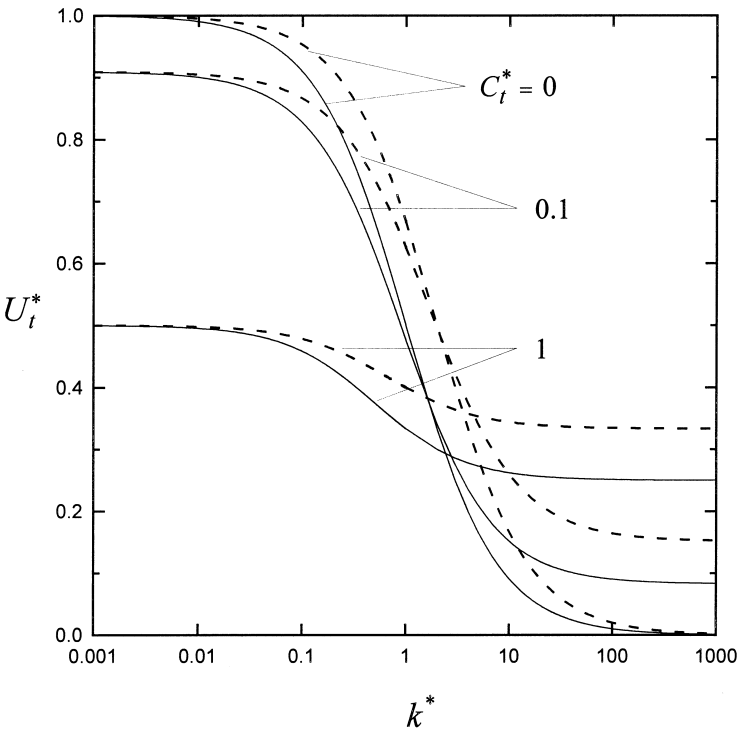

Fig. 2. Plots of the normalized thermophoretic velocity $U_{\mathrm{t}}^{*}(=$ $\left.\rho T_{0} U / C_{\mathrm{s}} \eta E_{\infty}\right)$ of a cylindrical particle (solid curves, as computed from Eq. (12)) and of a spherical particle (dashed curves, as computed from Eq. (14)) versus the conductivity ratio $k^{*}$ with $C_{\mathrm{t}}^{*}\left(=2 C_{\mathrm{m}}^{*}\right)$ as a parameter.

kinetic-theory values for complete thermal and momentum accommodations appear to be $C_{\mathrm{s}}=$ $1.17, C_{\mathrm{t}}=2.18$, and $C_{\mathrm{m}}=1.14$ [25]. The validity of the expression for the thermal creep velocity in Eq. (8b) is based on the assumption that the fluid is only slightly nonuniform in the undisturbed temperature on the length scale of the particle radius. The derivative $\partial T / \partial \phi$ can be evaluated from the temperature distribution given by Eq. (4a).

The general solution to Eq. (6) in the polar coordinate system $(\omega, \phi)$ is [26]:

$\Psi=\left(d_{1} \omega^{-1}+d_{2} \omega+d_{3} \omega \ln \omega+d_{4} \omega^{3}\right) \sin \phi$,

where the constants $d_{1}, d_{2}, d_{3}$ and $d_{4}$ are to be determined from boundary conditions Eq. (8a), Eq. (8c) and Eq. (8d) using Eq. (9). The result of the stream function is given by:

$\Psi=U a^{2} \frac{\sin \phi}{\omega}$,

which shows that the fluid flow around the cylinder undergoing thermophoretic motion is irrotational and exerts no drag force on the cylinder. It is well known that there exists no solution of the creepingflow equation (Eq. (6)) for the two-dimensional body-force-driven motion of an infinite-length circular cylinder in an unbounded viscous fluid (sometimes referred to as Stokes' paradox) [27]. In the case of thermophoresis, however, the hydrodynamic force on any boundary enclosing the particle is zero, since the temperature field produces no bulk (body) forces in the fluid. The existence of a solution of irrotational flow around the force-free thermophoretic cylinder in the form of Eq. (11) is due to the effect of thermal slip at the particle surface given by the third term on the right-hand side of Eq. (8b).

\section{$<$ sbm 42>2.3. Thermophoretic velocity}

Applying boundary condition (8b) together with (4a) and $(7(a, b))$ to Eq. (11), one obtains the thermophoretic velocity of the cylindrical particle as:

$U=\frac{C_{\mathrm{s}}\left(1+k^{*} C_{\mathrm{t}}^{*}\right)}{\left(1+2 C_{\mathrm{m}}^{*}\right)\left(1+k^{*}+k^{*} C_{\mathrm{t}}^{*}\right)} \frac{\eta E_{\infty}}{\rho T_{0}}$,

where:

$C_{\mathrm{m}}^{*}=C_{\mathrm{m}} l / a$.

According to Eq. (12), particles with large thermal conductivity and small Knudsen number (say, $k^{*}=100$ and $\left.l / a=0.01\right)$ will migrate at velocities of the order of $10^{-5} \mathrm{~m} \mathrm{~s}^{-1}$ with $E_{\infty}=10^{4} \mathrm{~K} \mathrm{~m}^{-1}$; such a temperature gradient is easily attainable in thermal boundary layers.

Values of the normalized thermophoretic velocity (or mobility) $U_{\mathrm{t}}^{*}\left(=\rho T_{0} U / C_{\mathrm{s}} \eta E_{\infty}\right)$ of the cylindrical particle evaluated from Eq. (12) with various values of $k^{*}, C_{\mathrm{t}}^{*}$, and $C_{\mathrm{m}}^{*}$ are plotted by the solid curves in Figs. 2 and 3 . The typical relation $C_{\mathrm{t}}=2 C_{\mathrm{m}}$ is chosen for the calculations because their reasonable values are so related. It is understood that the practical values of $C_{\mathrm{t}}^{*}$ and $C_{\mathrm{m}}^{*}$ are less than unity with the assumption of small Knudsen number. It can be found that $U_{\mathrm{t}}^{*}$ is a monotonic decreasing function of $k^{*}$ for given values of $C_{\mathrm{t}}^{*}$ and $C_{\mathrm{m}}^{*}$. The reason for this consequence is that a relatively high conductivity of the particle reduces the local temperature gradient (and thus, the thermal creep effect) along the particle surface. When the value of $k^{*}$ is large- 
(say, $>10$ ), $U_{\mathrm{t}}^{*}$ increases with the increase in $C_{\mathrm{t}}^{*}$ or $C_{\mathrm{m}}^{*}$ (or the decrease in the radius of the particle) for a fixed value of $k^{*}$; however, when the value of $k^{*}$ is small (say, $<1.5$ ), $U_{\mathrm{t}}^{*}$ decreases with the increase in $C_{\mathrm{t}}^{*}$ or $C_{\mathrm{m}}^{*}$ for a fixed value of $k^{*}$. This behavior is physically explainable: a large particle will enhance the effect of the relative conductivity of the particle on the thermophoretic mobility, while a small particle will lessen this effect. In the limiting case of $C_{\mathrm{t}}^{*}=0$ and $C_{\mathrm{m}}^{*}=0$, $U_{\mathrm{t}}^{*}=1$ as $k^{*}=0$ and $U_{\mathrm{t}}^{*}=0$ as $k^{*} \rightarrow \infty$.

For the corresponding thermophoresis of an aerosol sphere of radius $a$, Brock [15] obtained the analytical formula for the particle velocity,

$$
U=\frac{2 C_{\mathrm{s}}\left(1+k^{*} C_{\mathrm{t}}^{*}\right)}{\left(1+2 C_{\mathrm{m}}^{*}\right)\left(2+k^{*}+2 k^{*} C_{\mathrm{t}}^{*}\right)} \frac{\eta E_{\infty}}{\rho T_{0}},
$$

where $k^{*}, C_{\mathrm{t}}^{*}$, and $C_{\mathrm{m}}^{*}$ are still defined by Eq. (5ab) and Eq. (13). The normalized migration velocity $U_{t}^{*}$ of the thermophoretic sphere calculated from Eq. (14) for various values of $k^{*}, C_{\mathrm{t}}^{*}$, and $C_{\mathrm{m}}^{*}$ are drawn by the dashed curves in Figs. 2 and 3 for comparison. One can see that the behavior of the dependence of $U_{\mathrm{t}}^{*}$ on the parameters

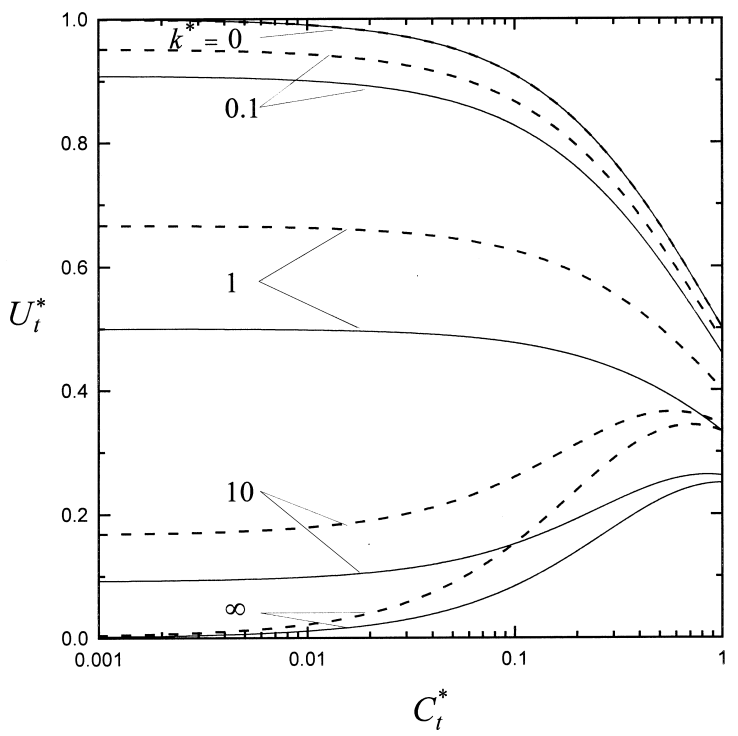

Fig. 3. Plots of the normalized thermophoretic velocity $U_{\mathrm{t}}^{*}(=$ $\left.\rho T_{0} U / C_{\mathrm{s}} \eta E_{\infty}\right)$ of a cylindrical particle (solid curves, as computed from Eq. (12)) and of a spherical particle (dashed curves, as computed from Eq. (14)) versus $C_{\mathrm{t}}^{*}\left(=2 C_{\mathrm{m}}^{*}\right)$ with $k^{*}$ as a parameter. $k^{*}, C_{\mathrm{t}}^{*}$ and $\mathrm{C}_{\mathrm{m}}^{*}$ for a cylindrical particle undergoing thermophoresis normal to its axis is quite similar to that for a thermophoretic sphere. For a set of given values of $k^{*}, C_{\mathrm{t}}^{*}$ and $C_{\mathrm{m}}^{*}$, the normalized thermophoretic velocity of a cylindrical particle is always smaller than that of a sphere (even in the limit $l / a=0$, or $C_{\mathrm{t}}^{*}=C_{\mathrm{m}}^{*}=0$ ), except for the limiting case of $k^{*}=0$, in which the normalized thermophoretic velocities are equal for both particle shapes. For the case of large $k^{*}$ and small $C_{\mathrm{t}}^{*}$, the cylinder has a thermophoretic mobility only about half of that of the sphere.

\section{Photophoresis of a cylinder}

We now consider the steady two-dimensional photophoretic motion of a long circular cylindrical particle in the direction normal to its axis. The incident light is imposed in the $x$ direction with intensity (incoming illumination energy flux) $I$. Again, the Knudsen number, Reynolds number, and Peclet number are assumed to be small and all the physical properties of the particle and the surrounding gas are taken to be constant. Our objective is to determine the photophoretic velocity of the cylinder.

\subsection{Temperature distribution}

The temperature distribution for the fluid is still governed by the Laplace equation (1a). However, instead of Eq. (1b), the temperature distribution inside the radiation absorbing particle is described by:

$\nabla^{2} \hat{T}=-\frac{1}{k_{\mathrm{p}}} Q(\omega, \phi)$

where $Q(\omega, \phi)$ is the volumetric thermal energy generation rate resulting from local radiation absorption. For a plane monochromatic wave, the function $Q(\omega, \phi)$ is related to the electric field $\mathbf{E}(\omega, \phi)$ inside the particle according to the Lorenz-Mie theory [14]:

$Q(\omega, \phi)=\frac{4 \pi v \kappa I}{\lambda} \frac{|\mathbf{E}(\omega, \phi)|^{2}}{\left|\mathbf{E}_{0}\right|^{2}}=\frac{4 \pi v \kappa I}{\lambda} B(\zeta, \phi)$. 
Here, $v$ and $\kappa$ are the real and imaginary parts of the complex refractive index $N(N=v+i \kappa)$ of the particle, $\mathbf{E}_{0}$ is the incident electric field strength, $\lambda$ is the wavelength of the incident radiation, $B(\zeta, \phi)$ is the dimensionless electric field distribution function, and $\zeta=\omega / a$.

The boundary conditions for the temperature field at the particle surface and inside the particle are still given by Eqs. $(3 \mathrm{a}-\mathrm{c})$. We further require that the fluid temperature far from the cylinder be unaffected by the cylinder's presence so that:

$\omega \rightarrow \infty: \quad T \rightarrow T_{\infty}$,

where $T_{\infty}$ is the temperature

of the bulk gas which is a constant now.

A sufficiently general solution to

Eqs. (1a) and (15) is:

$T=T_{\infty}+T_{\infty} \sum_{n=1}^{\infty}\left[A_{n} \cos (n \phi)+B_{n} \sin (n \phi)\right] \zeta^{-n}$,

$\hat{T}=T_{0}+T_{\infty} \sum_{n=1}^{\infty}\left\{\left[C_{n} \zeta^{n}+R_{n}(\zeta)\right] \cos (n \phi)\right.$

$\left.+\left[D_{n} \zeta^{n}+S_{n}(\zeta)\right] \sin (n \phi)\right\}$,

where:

$$
\begin{aligned}
& \left\{\begin{array}{l}
R_{n}(\zeta) \\
S_{n}(\zeta)
\end{array}\right\} \\
& =\frac{2 v \kappa a^{2} I}{n \hat{\lambda} \hat{\kappa} T_{\infty}}\left[\zeta^{n} \int_{\zeta}^{1} t^{-n+1} \int_{0}^{2 \pi} B(t, \phi)\left\{\begin{array}{c}
\cos (n \phi) \\
\sin (n \phi)
\end{array}\right\}\right. \\
& \times \mathrm{d} \phi \mathrm{d} t \\
& \left.+\zeta^{-n} \int_{0}^{\zeta} t^{n+1} \int_{0}^{2 \pi} B(t, \phi)\left\{\begin{array}{c}
\cos (n \phi) \\
\sin (n \phi)
\end{array}\right\} \mathrm{d} \phi \mathrm{d} t\right],
\end{aligned}
$$

and $T_{0}$ is the temperature at the axis of the cylinder. A solution of this form immediately satisfies boundary conditions (3c) and (17). The unknown coefficients $A_{\mathrm{n}}, B_{\mathrm{n}}, C_{\mathrm{n}}$ and $D_{\mathrm{n}}$ are to be determined using the boundary conditions at the particle surface. The derivation of solution (18b) is presented in Appendix A.
Applying the boundary conditions (3a) and (3b) along the surface of the cylinder to Eqs. (18a,b), we obtain:

$$
\begin{aligned}
& A_{n}=\frac{k^{*}\left[n R_{n}(1)-R_{n}^{\prime}(1)\right]}{n+n k^{*}+n^{2} k^{*} C_{\mathrm{t}}^{*}}, \\
& B_{n}=\frac{k^{*}\left[n S_{n}(1)-S_{n}^{\prime}(1)\right]}{n+n k^{*}+n^{2} k^{*} C_{\mathrm{t}}^{*},} \\
& C_{n}=\frac{\left(n k^{*}+n^{2} k^{*} C_{\mathrm{t}}^{*}\right) R_{n}^{/}(1)-R_{n}(1)}{1+n^{2} k^{*}+n^{3} k^{*} C_{\mathrm{t}}^{*}}, \\
& D_{n}=\frac{\left(n k^{*}+n^{2} k^{*} C_{\mathrm{t}}^{*}\right) S_{n}^{\prime}(1)-S_{n}(1)}{1+n^{2} k^{*}+n^{3} k^{*} C_{\mathrm{t}}^{*}},
\end{aligned}
$$

where $k^{*}$ and $C_{\mathrm{t}}^{*}$ have been defined by Eqs. $(5 \mathrm{a}, \mathrm{b})$ and the prime on $R_{\mathrm{n}}(\zeta)$ and $S_{\mathrm{n}}(\zeta)$ means differentiation with respect to $\zeta$. Thus, the temperature distribution expressed by Eqs. $(18 \mathrm{a}, \mathrm{b})$ is completely solved.

\section{$<$ sbm 42>3.2. Fluid velocity distribution}

Having determined the temperature distribution, we can begin finding out the fluid velocity field. The fluid motion generated by the photophoretic migration of the cylinder is still governed by the Stokes equation (Eq. (6)) and subject to the boundary conditions $8 \mathrm{a}-\mathrm{d}$. But now, the derivative $\partial T / \partial \phi$ in Eq. (8b) should be evaluated from the temperature distribution given by Eq. (18a).

It can be shown that, similar to the case of thermophoresis considered in the previous section, the fluid flow around the force-free cylinder undergoing photophoretic motion is also irrotational and described by the stream function in the form of Eq. (11).

\subsection{Photophoretic velocity}

Using Eqs. (7a,b) and (18a) and applying boundary condition (8b) to Eq. (11), we obtain the photophoretic velocity of the cylinder,

$U=$

$-\frac{C_{\mathrm{s}} J_{1}}{2\left(1+2 C_{\mathrm{m}}^{*}\right)\left(1+k^{*}+k^{*} C_{\mathrm{t}}^{*}\right)} \frac{\eta I}{k \rho T_{\infty}}$.

Here, $J_{1}$ is the so-called photophoretic asymmetry factor [21], 


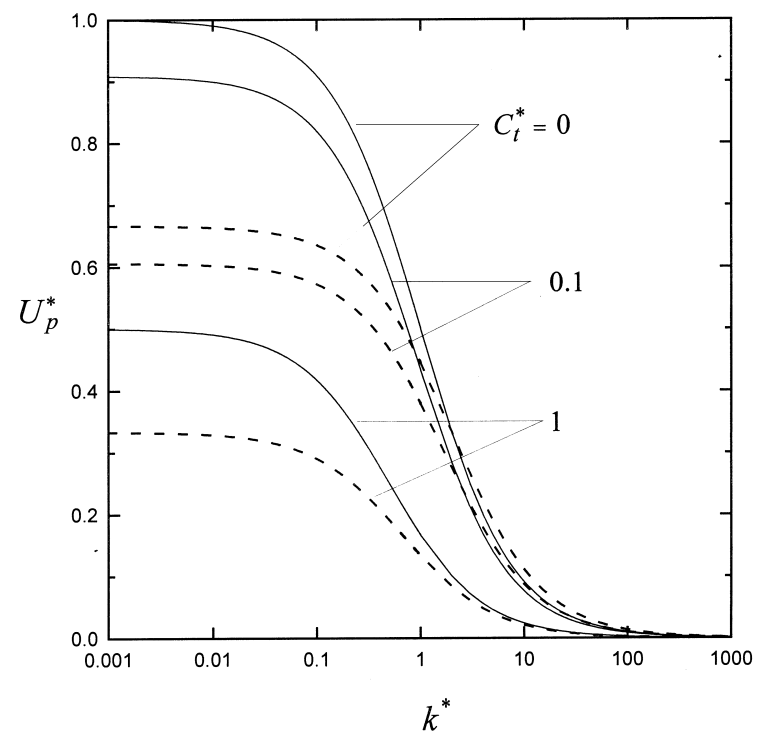

Fig. 4. Plots of the normalized photophoretic velocity $U_{\mathrm{p}}^{*}(=$ $\left.-2 k \rho T_{\infty} U / C_{\mathrm{s}} J_{1} \eta I\right)$ of a cylindrical particle (solid curves, as computed from Eq. (21)) and of a spherical particle (dashed curves, as computed from Eq. (24)) versus the conductivity ratio $k^{*}$ with $C_{\mathrm{t}}^{*}\left(=2 C_{\mathrm{m}}^{*}\right)$ as a parameter.

$J_{1}=\frac{4 v \kappa a}{\lambda} \int_{0}^{1} \int_{0}^{2 \pi} B(\zeta, \phi) \zeta^{2} \cos \phi \mathrm{d} \phi \mathrm{d} \zeta$,

which depends on the complex refractive index $(N=v+i \kappa)$ and the normalized size $(2 \pi a / \lambda)$ of the particle. The asymmetry factor represents a weighted integration of the heat source function over the particle volume and defines the sign (and the magnitude) of the photophoretic force. If $J_{1}<0$, the particle moves in the direction of the light beam (positive photophoresis). If $J_{1}>0$, the particle moves in the opposite direction (negative photophoresis). For a completely opaque cylindrical particle the heat sources are concentrated on the illuminated part of the particle surface, namely,

$B(\zeta, \phi)=\left\{\begin{array}{cc}\frac{-\lambda}{2 \pi v \kappa a} \cos \phi \delta(\zeta-1) & \text { for } \frac{\pi}{2} \leq \phi \leq \frac{3 \pi}{2} \\ 0 & \text { otherwise, }\end{array}\right.$

where $\delta(\zeta-1)$ is a Dirac delta function which equals infinity if $\zeta=1$ and vanishes otherwise. The substitution of Eq. (23) into Eq. (22) results in $J_{1}=-1 / 2$ knowing that $\int_{0}^{1} g(\zeta) \delta(\zeta-1) d \zeta=(1 /$ 2) $g(1)$. It is obvious that the range of the asymmetry factor is $-1 / 2 \leq J_{1} \leq 1 / 2$. According to Eq. (21), the photophoretic velocity at illumination of an intensity comparable with the solar constant $\left(1353 \mathrm{~W} \mathrm{~m}^{-2}\right)$ is of the order of $10^{-5} \mathrm{~m} \mathrm{~s}^{-1}$.

The corresponding photophoresis of an aerosol sphere of radius $a$ was analytically studied by Reed [16] and Mackowski [14]. The photophoretic velocity of the sphere can be expressed as:

$U=$

$-\frac{2 C_{s} J_{1}}{3\left(1+2 C_{\mathrm{m}}^{*}\right)\left(2+k^{*}+2 k^{*} C_{\mathrm{t}}^{*}\right)} \frac{\eta I}{k \rho T_{\infty}}$.

Here, the photophoretic asymmetry factor $J_{1}$ becomes:

$J_{1}=\frac{6 \pi v \kappa a}{\lambda} \int_{0}^{1} \int_{0}^{\pi} B(\xi, \theta) \xi^{3} \cos \theta \sin \theta \mathrm{d} \theta \mathrm{d} \xi$,

where $\xi=r / a,(r, \theta, \phi)$ is the spherical coordinates measured from the center of the particle, and the dimensionless electric field distribution function $B(\xi, \theta)$ is related to the volumetric energy generation rate $Q(r, \theta)$ through a formula in the form of Eq. (16). For a completely opaque sphere of radius $a$,

$B(\xi, \theta)=\left\{\begin{array}{cc}\frac{-\lambda}{2 \pi v \kappa a} \cos \theta \delta(\xi-1) & \text { for } \frac{\pi}{2} \leq \theta \leq \pi \\ 0 & \text { for } 0 \leq \theta \leq \frac{\pi}{2} .\end{array}\right.$

Again, for this situation, Eq. (25) and Eq. (26) lead to $J_{1}=-1 / 2$.

Numerical values of the normalized photophoretic velocity (or mobility) $U_{\mathrm{p}}^{*} \quad(=-$ $\left.2 k \rho T_{\infty} U / C_{\mathrm{s}} J_{1} \eta I\right)$ of a cylindrical particle, calculated from Eq. (21) for various values of $k^{*}$, $C_{\mathrm{t}}^{*}$ and $C_{\mathrm{m}}^{*}$ are plotted by solid curves in Figs. 4 and 5. The corresponding results for the photophoresis of an aerosol sphere given by Eq. (24) are also drawn by dashed curves in the same figures for comparison. For both particle shapes, $U_{\mathrm{p}}^{*}$ decreases monotonically with increasing $k^{*}$ for fixed values of $C_{\mathrm{t}}^{*}$ and $C_{\mathrm{m}}^{*}$ and with increasing $C_{\mathrm{t}}^{*}$ or $C_{\mathrm{m}}^{*}$ (or decreasing radius of the particle) for a given value of $k^{*}$. In the limiting case of 
$k^{*} \rightarrow \infty, U_{\mathrm{p}}^{*}=0$. For a set of fixed values of $k^{*}$, $C_{\mathrm{t}}^{*}$ and $C_{\mathrm{m}}^{*}$, the normalized migration velocity of a cylindrical particle undergoing photophoresis normal to its axis is greater than that of a photophoretic sphere if $C_{\mathrm{t}}^{*} \geq 1 / 2$ or if $C_{\mathrm{t}}^{*}<1 / 2$ but $k^{*}<2 /\left(1-2 C_{\mathrm{t}}^{*}\right)$, and is smaller than that of a photophoretic sphere if $C_{\mathrm{t}}^{*}<1 / 2$ and $k^{*}>2 /(1-$ $\left.2 C_{\mathrm{t}}^{*}\right)$. Of course, when $C_{\mathrm{t}}^{*}<1 / 2$ and $k^{*}=2 /(1-$ $\left.2 C_{\mathrm{t}}^{*}\right)$, the cylindrical and spherical particles will have identical normalized photophoretic velocity for constant values of $k^{*}, C_{\mathrm{t}}^{*}$, and $C_{\mathrm{m}}^{*}$. For the case of small $k^{*}$, the cylinder can have a photophoretic mobility about $50 \%$ larger than that of the sphere. For the case of large $k^{*}$ and small $C_{\mathrm{t}}^{*}$ however, the photophoretic mobility of the cylinder is about $25 \%$ less than that of the sphere.

\section{Conclusions}

Two problems of similar physical and mathematical structures are analytically studied in this work: the thermophoresis of a cylindrical particle

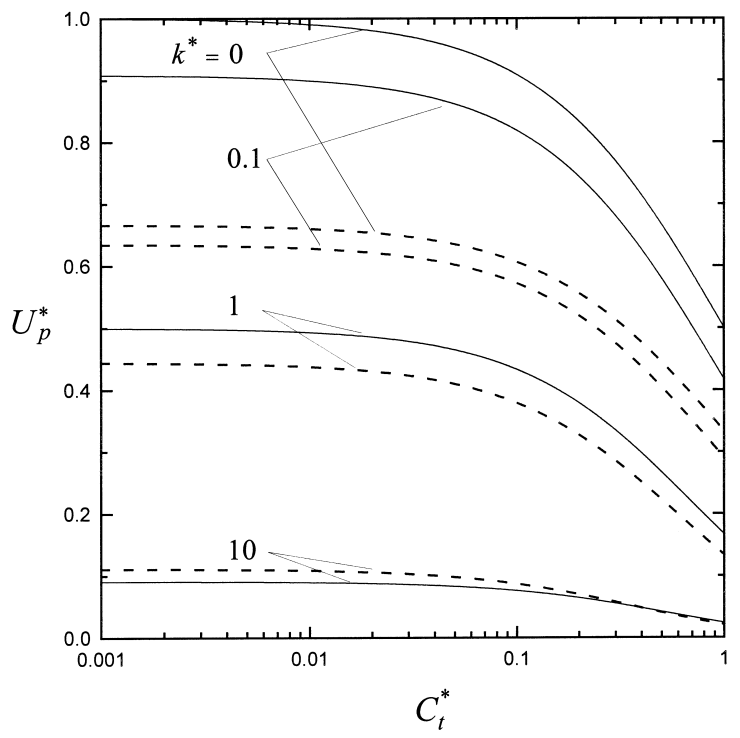

Fig. 5. Plots of the normalized photophoretic velocity $U_{\mathrm{p}}^{*}(=$ $\left.-2 k \rho T_{\infty} U / C_{\mathrm{s}} J_{1} \eta I\right)$ of a cylindrical particle (solid curves, as computed from Eq. (21)) and of a spherical particle (dashed curves, as computed from Eq. (24)) versus $C_{\mathrm{t}}^{*}\left(=2 C_{\mathrm{m}}^{*}\right)$ with $k^{*}$ as a parameter. normal to its axis in a nonisothermal gaseous medium and the photophoresis of an aerosol cylinder normal to its axis illuminated by a beam of light of sufficient intensity. It is assumed that the Knudsen number is low so that the fluid flow is in the continuum regime. Under the condition of vanishingly small Peclet and Reynolds numbers, the temperature distribution inside and outside the particle and the flow field for the surrounding fluid are obtained with taking account of the effects of temperature jump, thermal slip, and frictional slip at the gas/particle interface. Closed-form expressions for the thermophoretic and photophoretic velocities of the cylindrical particle as functions of relevant parameters are obtained in Eq. (12) and Eq. (21), respectively. Compared with an aerosol sphere under the same condition, the cylindrical particle always has a smaller thermophoretic mobility, but can have a greater or smaller photophoretic mobility, depending on the relative magnitudes of the temperature jump coefficient and of the thermal conductivities of the gas-particle system.

Because a simple analytical solution is not feasible in prolate and oblate spheroidal coordinate systems if the temperature jump or frictional slip at the particle surface is included [22], it is a formidable task to obtain analytical formulas for the thermophoretic or photophoretic velocity of a spheriodal particle as a function of $C_{\mathrm{t}}^{*}$ and $C_{\mathrm{m}}^{*}$. Therefore, our analysis for a circular cylindrical particle should be worthwhile in understanding the effects of particle shape on thermophoresis and photophoresis.

\section{Acknowledgements}

Part of this research was supported by the National Science Council of the Republic of China.

\section{Appendix A. Derivation of Eq. (18b)}

For conciseness the derivation of Eqs. (18b) and (19) is given here. If we define dimensionless quantities $\bar{T}=\left(\hat{T}-T_{0}\right) / T_{\infty}, \quad \zeta=\omega / a$, and $g=$ $a^{2} Q / \hat{k} T_{0}$, then Eq. (15) becomes: 
$\zeta \frac{\partial}{\partial \zeta}\left(\zeta \frac{\partial \bar{T}}{\partial \zeta}\right)+\frac{\partial^{2} \bar{T}}{\partial \phi^{2}}=-\zeta^{2} g(\zeta, \phi)$

The solution to Eq. (A1) can be taken as the sum of the homogeneous and particular solutions, denoted by $T_{1}$ and $T_{2}$, respectively. The homogeneous solution that is valid at the axis of the cylindrical particle is:

$T_{1}=\sum_{n=1}^{\infty} \zeta^{n}\left[C_{n} \cos (n \phi)+D_{n} \sin (n \phi)\right]$

where $C_{n}$ and $D_{n}$ are undetermined coefficients. The particular solution $T_{2}$ can be obtained from a transformation of Eq. (A1) into ordinary differential equations. Multiplying Eq. (A1) through by $\cos (n \phi) \mathrm{d} \phi$ and $\sin (n \phi) \mathrm{d} \phi$, respectively, and integrating from 0 to $2 \pi$ leads to:

$\zeta \frac{\partial}{\partial \zeta}\left(\zeta \frac{\partial R}{\partial \zeta}\right)-n^{2} R_{n}=-g_{n}$

$\zeta \frac{\partial}{\partial \zeta}\left(\zeta \frac{\partial S_{n}}{\partial \zeta}\right)-n^{2} S_{n}=-h_{n}$

where:

$R_{n}(\zeta)=\frac{1}{\pi} \int_{0}^{2 \pi} T_{2}(\zeta, \phi) \cos (n \phi) \mathrm{d} \phi$,

$S_{n}(\zeta)=\frac{1}{\pi} \int_{0}^{2 \pi} T_{2}(\zeta, \phi) \sin (n \phi) \mathrm{d} \phi$,

and

$g_{n}(\zeta)=\frac{1}{\pi} \zeta^{2} \int_{0}^{2 \pi} g(\zeta, \phi) \cos (n \phi) \mathrm{d} \phi$,

$h_{n}(\zeta)=\frac{1}{\pi} \zeta^{2} \int_{0}^{2 \pi} g(\zeta, \phi) \sin (n \phi) \mathrm{d} \phi$.

Obviously, Eqs. (A4a,b) yield:

$T_{2}=R_{n}(\zeta) \cos (n \phi)+S_{n}(\zeta) \sin (n \phi)$.

The solution to Eqs. (A3a,b) can be obtained by the method of variation of parameters, with the result:

$$
\begin{aligned}
R_{n}= & \frac{1}{2 n} \zeta^{n} \int_{\zeta}^{1} t^{-n-1} \cdot g_{n}(t) \mathrm{d} t \\
& +\frac{1}{2 n} \zeta^{-n} \int_{0}^{\zeta} t^{n-1} \cdot g_{n}(t) \mathrm{d} t,
\end{aligned}
$$

$$
\begin{aligned}
S_{n}= & \frac{1}{2 n} \zeta^{n} \int_{\zeta}^{1} t^{-n-1} \cdot h_{n}(t) \mathrm{d} t \\
& +\frac{1}{2 n} \zeta^{-n} \int_{0}^{\zeta} t^{n-1} \cdot h_{n}(t) \mathrm{d} t .
\end{aligned}
$$

The combination of Eq. (A2) and (A6) results in Eq. (18b) and the substitution of Eqs. (A5a,b) into Eqs. (A7a,b) gives Eq. (19).

\section{Appendix B. Nomenclature}

$a$ particle radius (m)

$B$ dimensionless electric field distribution function defined by Eq. (16)

$C_{\mathrm{m}} \quad$ dimensionless coefficient accounting for the frictional slip

$C_{\mathrm{m}}^{*} \quad$ dimensionless parameter defined by Eq. (13)

$C_{\mathrm{s}}$ dimensionless coefficient accounting for the thermal slip

$C_{\mathrm{t}}$ dimensionless coefficient accounting for the temperature jump

$C_{\mathrm{t}}^{*} \quad$ dimensionless parameter defined by Eq. (5b)

$\mathbf{e}_{x} \quad$ unit vector in $x$ direction

$E_{\infty} \quad$ prescribed temperature gradient in the absence of the particle $\left(\mathrm{K} \mathrm{m}^{-1}\right)$

$I$ intensity of the incident light beam (W $\mathrm{m}^{-2}$ )

$J_{1} \quad$ the photophoretic asymmetry factor of the particle

$k$ thermal conductivity of the fluid (W $\mathrm{m}^{-1} \mathrm{~K}^{-1}$ )

$k_{\mathrm{p}} \quad$ thermal conductivity of the particle (W $\mathrm{m}^{-1} \mathrm{~W}^{-1}$ )

$k^{*} \quad$ ratio of thermal conductivities between the particle and the fluid

$l$ mean free path of the gas molecules (m)

$Q \quad$ the volumetric thermal energy generation rate inside the particle $\left(\mathrm{W} \mathrm{m}^{-3}\right)$

$r \quad$ radial spherical coordinate $(\mathrm{m})$

$T$ temperature distribution in the fluid phase $(\mathrm{K})$

$\hat{T} \quad$ temperature distribution inside the particle $(\mathrm{K})$

$T_{0} \quad$ absolute temperature at the center of the particle $(\mathrm{K})$ 
$T_{\infty} \quad$ bulk-gas absolute temperature in the absence of the particle $(\mathrm{K})$

$U$ migration velocity of the particle (m $\left.\mathrm{s}^{-1}\right)$

$U_{\mathrm{p}}^{*} \quad$ normalized photophoretic velocity of the particle $\left(=-2 k \rho T_{\infty} U / C_{\mathrm{s}} J_{1} \eta I\right)$

$U_{\mathrm{t}}^{*} \quad$ normalized thermophoretic velocity of the particle $\left(=\rho T_{0} U / C_{\mathrm{s}} \eta E_{\infty}\right)$

$v_{\omega}, v_{\phi}$ components of the fluid velocity in polar coordinates $\left(\mathrm{m} \mathrm{s}^{-1}\right)$

\section{Greek letters}

$\zeta \quad$ dimensionless radial polar coordinate $(=$ $\omega / a)$

$\eta \quad$ viscosity of the fluid $\left(\mathrm{kg} \mathrm{m}^{-1} \mathrm{~s}^{-1}\right)$

$\theta, \phi$ angular spherical coordinates

$\kappa \quad$ imaginary part of the complex refractive index of the particle

$\lambda$ the wavelength of the incident light beam (m)

$v \quad$ real part of the complex refractive index of the particle

$\xi \quad$ dimensionless radial spherical coordinate $(=r / a)$

$\rho \quad$ density of the fluid $\left(\mathrm{kg} \mathrm{m}^{-3}\right)$

$\phi \quad$ angular polar coordinate

$\Psi \quad$ stream function of the fluid flow $\left(\mathrm{m}^{2} \mathrm{~s}^{-1}\right)$

$\omega$ radial polar coordinate $(\mathrm{m})$

\section{References}

[1] J.C. Maxwell, Phil. Trans. R. Soc. 170 (1879) 231.
[2] E.H. Kennard, Kinetic Theory of Gases, McGraw-Hill, New York, 1938, p. 291.

[3] A.G.B.M. Sasse, W.W. Nazaroff, A.J. Gadgil, Aerosol Sci. Technol. 20 (1994) 227.

[4] N. Montassier, D. Boulaud, A. Renoux, J. Aerosol Sci. 22 (1991) 677.

[5] M.C. Weinberg, J. Am. Ceram. Soc. 65 (1982) 81.

[6] Y. Ye, D.Y.H. Pui, B.Y.H. Liu, S. Opiolka, S. Blumhorst, H. Fissan, J. Aerosol Sci. 22 (1991) 63.

[7] M.M.R. Williams, J. Phys. D Appl. Phys. 19 (1986) 1631.

[8] O. Preining, in: C.N. Davies (Ed.), Aerosol Science, Academic Press, New York, 1966, p. 111.

[9] S. Arnold, M. Lewittes, J. Appl. Phys. 53 (1982) 5314.

[10] W.M. Greene, R.E. Spjut, E. Bar-Ziv, A.F. Sarofim, J.P. Longwell, J. Opt. Soc. Am. B 2 (1985) 998.

[11] V. Chernyah, S. Beresnev, J. Aerosol Sci. 24 (1993) 857.

[12] C. Orr, E.Y.H. Keng, J. Atmos. Sci. 21 (1964) 475.

[13] M. Kerker, D.D. Cooke, J. Opt. Soc. Am. 72 (1982) 1267.

[14] D.W. Mackowski, Int. J. Heat Mass Transf. 32 (1989) 843.

[15] J.R. Brock, J. Colloid Sci. 17 (1962) 768.

[16] L.D. Reed, J. Aerosol Sci. 8 (1977) 123.

[17] L. Waldmann, K.H. Schmitt, in: C.N. Davies (Ed.), Aerosol Science, Academic Press, New York, 1966, p. 137.

[18] P.J. Whitmore, J. Aerosol Sci. 12 (1981) 1.

[19] S. Beresnev, V. Chernyah, Phys. Fluids 7 (1995) 1743.

[20] G.M. Hidy, J.R. Brock, J. Geophys. Res. 72 (1967) 455.

[21] Yu.I. Yalamov, V.B. Kutukov, E.R. Shchukin, J. Colloid Interface Sci. 57 (1976) 564.

[22] K.H. Leong, J. Aerosol Sci. 15 (1984) 511.

[23] D.W. Mackowski, J. Colloid Interface Sci. 140 (1990) 138.

[24] W. Zulehner, H. Rohatschek, J. Colloid Interface Sci. 26 (1995) 201.

[25] L. Talbot, R.K. Cheng, R.W. Schefer, D.R. Willis, J. Fluid Mech. 101 (1980) 737.

[26] A.B. Basset, A Treatise on Hydrodynamics, vol. 2, Dover, New York, 1961, p. 260.

[27] J. Happel, H. Brenner, Low Reynolds Number Hydrodynamics, Martinus Nijhoff, Amsterdam, 1983. 